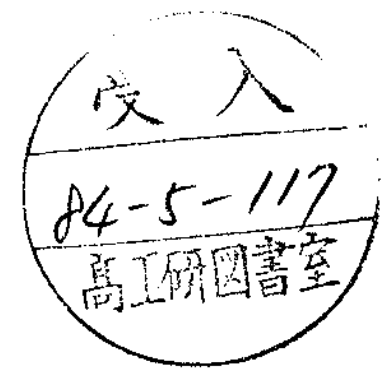

EUROPEAN ORGANIZATION FOR NUCLEAR RESEARCH

CERN/EP 84-37

23 March 1984

\title{
AN INVESTIGATION OF THE EMC EFEECT USING ANTINEUTRINO INTERACTIONS IN DEUTERIUM AND NEON
}

A.M. Cooper ${ }^{1}$, J. Derkaoui ${ }^{3}$, M.L. Faccini-Turluer ${ }^{6}$, M.A. Parker ${ }^{1}$, A. Petridis ${ }^{14}$, R.A. Sansumis, C. Valléel3, G.W. van Apeldoorn ${ }^{1}$

M. Aderholz15, D. Allasia , T. Azemoon'19, A. Baldinis, K.W.J. Barnham ${ }^{14}$, J.H. Bartley 19 , D. Bertrand ${ }^{10(*)}$, F. Bobisut 4 , B. Brisson ${ }^{33}$,

F.W. Bullock 19 , M. Calicchio, P. Capiluppi ${ }^{3}$, E.F. Clayton ${ }^{14}$, L. Deck ${ }^{15}$, P.J. Fitch ${ }^{9}$, V. Flaminios, M.T. Fogli-Muciaccia , G. Gerbier ${ }^{3}$, J.G.V. Guy ${ }^{17}$, P.O. Hulth $11\left(*^{\frac{1}{x}}\right)$, H. Huzita $a^{4}$, G.T. Jones, P. Kasper ${ }^{7}$, H. Klein ${ }^{1}, C$. Kochowski ${ }^{18}$, P. Maragelo(***), A. Marzari-Chiesa ${ }^{7}$, R.P. Middleton ${ }^{9}$, S. Natali ${ }^{8}$, M. Neveu' ${ }^{18}$, S.W.O. Neale ${ }^{9}$, R. Pazzi ${ }^{5}$, P. Petiaul3, F. Romano ${ }^{8}$, A. Romero', A.M. Rossi ${ }^{3}$, F. Ruggieri', 3. Sacton ${ }^{10}$, N. Schmitz's, E. Simopouloul2, A. Tenner ${ }^{12}$ K. Varvel1 16 , A. Vayaki ${ }^{2}, W$. Venus ${ }^{17}$, D. Vignaud ${ }^{7}$, O. Villalobos-Baillie ${ }^{14}$, H. Wachsmuthil, J. Wel1si6, K.L. Wernhard ${ }^{15}(+)$, and R. Wigmans

\section{WA2S Collaboration}

NIKHEF-H, Amsterdam, The Netherlands.

Institute of Physics, University of Bergen, Norway.

Istit. di Fisica dell'Univ. di Bologna \& INFN Sezione di Bologna, Italy. Dipart. di Fisica de11'Univ. di Padova \& INFN Sezione di Padova, Italy. Istituto di Fisica dell'Università di Pisa \& INFN Sezione di Pisa, Italy. Département de Physique des Particules Elémentaires, CEN Saclay, France. Istit. di Fisica dell'Univ. di Torino \& INFN Sezione di Torino, Italy.

\section{WA59 Co11aboration}

- Istituto di Fisica dell'Universita e Sezione INFN, Bari, Italy.

9 University of Birmingham, U.K. Inter University Institute for High Energies ULB-VUB, Brussels, Belgium. CERN, European Organization for Nuclear Research, Geneva, Switzerland. Nuclear Research Centre Demokritos, Athens, Greece. LPNHE, Ecole Polytechnique, Palaiseau, France. Imperial College of Science and Technology, London, U.K. Max Planck Institut für Physik und Astrophysik, Munich, Germany. Department of Nuclear Physics, Oxford, U.K. Rutherford Appleton Laboratory, Chilton, Didcot, U.K. D.Ph. PE Centre d'Etudes Nucléaires, Saclay, France. University College London, U.K.

\section{Submitted to Physics Letters B}

(*) Qualified scientist, FNRS Belgium

(**) Now at Institute of Physics, University of Stockholm, Sweden

(***) Fellow at IRSIA, Belgium

$(+)$ Now at Siemens, Munich 


\section{ABSTRACT}

Antineutrino interactions in BEBC are compared to look for differences between the differential cross section ratios per nucleon in neon and in deuterium. The identical geometries, beam spectra and muon identification criteria and acceptances allow comparison with very small systematic errors. The results are compared in detail with $\mu$ and e scattering data from EMC and SLAC. We find no rise in the ratio do/dx $(\vec{v} \mathrm{Ne}) / \mathrm{do} / \mathrm{dx}\left(\bar{v} \mathrm{D}_{2}\right)$ at low $x$, independent of $Q^{2}$ up to $\left\langle Q^{2}\right\rangle \sim 14 \mathrm{GeV}^{2}$. 


\section{INTRODUCTION}

Recent results from the EMC [1] and Rochester-MIT-SLAC [2] groups have indicated that the nucleon structure functions measured using heavy nuclei and using deuterium are different. These results are illustrated in $\mathrm{fig}$. 1. At large $x(x>0.6)$ the SLAC data show the expected effect of Fermi motion [3]. At medium $x(0.3<x<0.6)$ both groups see a new effect which varies logarithmically with $A$ and seems independent of $Q^{2}$. At small $x(x<0.3)$ the effects seen by the two groups differ, suggesting $Q^{2}$ dependence in this $x$ range. The medium-x and small-x effects defy interpretation in terms of Fermi motion and have caused renewed interest in the problems of additivity and identity of nucleons in nuclei and in the application of QCD to nuclear physics.

Present mode1s [4] give varying predictions for the $A$ and $Q^{2}$ dependence of the effects, thus it is of interest to make comparisons with different targets and different $Q^{2}$ ranges. Our data covers a larger $Q^{2}$ range than the SLAC data, thus giving new information on $Q^{2}$ dependence at small $\mathrm{x}$. Also antineutrinos are the ideal tool to investigate current explanations of the effect which imply that the sea quark content increases in heavy nuclei, $[4 \mathrm{~d}, e, g, h, i, j, k, l, m, 0, q]$. The sea contributes strongly to the $\bar{v}$ cross section, so that an increase in sea would result in strong correlated changes in the $x$ and $y$ distributions. Previous comparisons using $v$ and $\bar{v}$ data in bubble chambers have less statistical [5] and systematic [6] precision and did not exploit the information in the $y$ and $Q^{2}$ distributions.

\section{EXPERIMENTAL TECHNIQUE}

This paper compares data from two exposures of BEBC, filled with $\mathrm{D}_{2}$ (WA25) and a 75 mole per cent $\mathrm{Ne}-\mathrm{H}_{2}$ mixture (WA59) respectively, to the same CERN-SPS wide band antineutrino beam. The two data sets were 
entirely reanalysed for this investigation, so that every possible source of systematic error was either eliminated or corrected. To ensure that the geometries, beam spectra and muon identification criteria and acceptances were identical:

(a) Events were required to 1 ie in the overlap of the two fiducial volumes used by the two experiments.

(b) Identical beam conditions were selected in the two runs. Beam constancy throughout both runs was ensured with the aid of the array of muon flux detectors distributed throughout the shielding [7]: all pulses for which the shape of the muon flux distribution was non-standard were excluded from the analysis.

(c) Charged Current (CC) events were identified using the same External Muon Identifier (EMI). The performance of each wire in the EMI was checked regularly using cosmic rays. Using only wires with high efficiencies throughout both runs, the data from the two experiments were reprocessed through the same muon identification program. All events were required to have muon momentum greater than $5 \mathrm{GeV} / \mathrm{c}$.

Corrections were then applied for:

(a) The background from Neutral Current (NC) events accidentally identified as CC events, which is different in the two liquids because their hadronic interaction lengths differ. This correction involves less than $1 \%$ of events.

(b) The difference in scan efficiency between the two experiments, which was corrected for separately for each topology. To avoid large corrections for the one-prong lopology, all one-prong events were removed from both samples. This leaves only a smaller correction (3\%) to the neon sample due to topology-changing reinteractions. This was estimated by comparing the hadronic showers in neon and deuterium and by analysing events with unbalanced charge (e.g. $u N \rightarrow \mu^{+}+$single positive hadron) which can only be produced by reinteraction processes. 


\section{METHOD OF COMPARISON WITH $e_{2} \mu$ DATA}

In principle, because of the additional contribution from the structure function $x_{3}$ to the cross section, $v$ or $v$ data can only be compared with $e$ or $\mu$ data using a model.

(A) The simplest models assume that the effect does not depend on $y$ or $Q^{2}$ or on whether a sea or valence quark was struck, and that the $x$ dependence is represented by the SLAC 1 ine or the EMC 1 ine illustrated in $\mathrm{fig}$. 1 .

(B) To account for the $x F_{3}$ term we use a simple quark parton model (QPM) based on the many interpretations $[4 \mathrm{~d}, e, \mathrm{~g}, \mathrm{i}, \mathrm{k}, \mathrm{m}, \mathrm{q}]$ of the EMC effect which ascribe the small $x$ and medium $x$ effects to an increase in the sea quark content and a softening of the valence quark momentum distribution in a heavy nucleus, respectively. We implement this model by parametrizing the structure functions $F_{2}$ and $\mathrm{xF}_{3}$ in terms of valence and sea quark momentum distributions varying as $x^{\alpha}(1-x)^{\beta}$ and $\delta(1+\gamma)(1-x)^{\gamma}$ respectively. For $D_{2}$ we use the parameter set of Allasia et al. $[8]^{(*)}$. For the heavy nuclei we change only:

(a) $\beta$ to $\beta+\Delta \beta$, to allow the valence quark distributions to soften and

(b) $\delta$ to $\mathrm{R}_{\mathrm{S}} \delta$ to allow the sea content to increase.

Clearly the values $\Delta \beta=0, R_{s}=1$ correspond to no nuclear effects. Applying this model to muoproduction we find that the values $\Delta \beta=0.2$, $R_{S}=1.35$ give the QPM 1 ine in $f i g .1$, which interpolates well through EMC data. If the amount and shape of the sea in $D_{2}$ are varied to account for the higher $Q^{2}$ of the EMC data, the corresponding value of $R_{s}$ varies only in the range 1.3 to 1.4 .

(*) Other parameter sets $[5,9]$ have been tried and the model is insensitive to this input. 


\section{TOTAL CROSS SECTION RATIO}

After the corrections detailed in sect. 2 have been made the event samples correspond to $4899 \mathrm{D}_{2}$ events and 12533 Ne events. Using the densities of deuterium and neon, and the muon fluxes $\phi_{\mu}$ (which are proportional to the $\bar{v}$ fluxes $\phi_{\bar{v}}$ ) recorded by identical detectors in the two experiments, and summed over all frames used, we obtain the total cross section ratio per nucleon

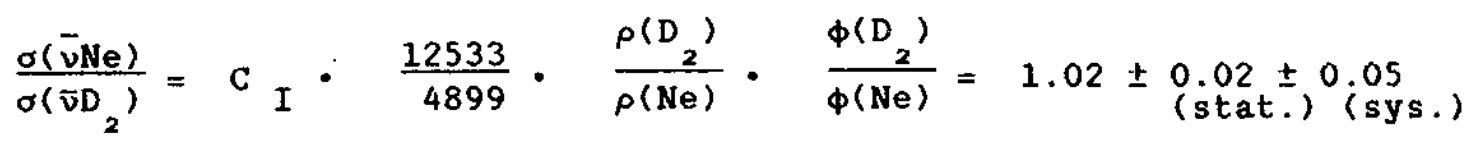

where $C_{I}=0.99$ is a correction factor for the non-isoscalarity of the 75 mole per cent $\mathrm{Ne} / \mathrm{H}_{2}$ mixture. The dominant systematic error arises from corrections on low topology event numbers. If we exclude events with total hadronic energy $\nu<2 \mathrm{GeV}$ we obtain

$$
\frac{\sigma(\bar{v} \mathrm{Ne})}{\sigma\left(\bar{v} D_{2}\right)}=1.04 \pm 0.02 \pm 0.03
$$

This value of the cross section ratio may be compared with the values of $1.07,0.97,1.11$ from the EMC, SLAC and QPM parametrizations discussed in the previous paragraph.

5. DIFFERENTIAL CROSS SECTIONS: MUON VARIABLES

We first compare the distributions of the muon variables

$$
v^{\mu}=\left(E-p_{L}\right)^{\mu} / M=x y,
$$

and

$$
\mathrm{p}_{\mathrm{T}}^{\mu} \approx \sqrt{\mathrm{x} \text { y } M\left(2 \mathrm{E}_{\bar{v}}(1-\mathrm{y})-\mathrm{M} \times \mathrm{y}\right)}
$$

which are well measured, so that systematic errors in the ratio of their distributions for $\mathrm{Ne}$ and $\mathrm{D}_{2}$ targets are small. The results are shown in fig. 2 and Table 1. The statistical and systematic errors, and the average $x$ and $Q^{2}$ values relevant to each bin, are also presented in table 1 . 
The lines in fig. 2 represent the application of the models described in sect. 3 to the muon variables accounting for (a) the energy spectrum of our beam (b) our acceptance and cuts and (c), in the case of the QPM model, the different couplings in $\bar{v}$ and $e / \mu$ processes. Since there is a systematic normalisation uncertainty both for our data (3-5\%) and the muon data $(7 \%)$, we allow the normalisation of these lines to be free when making $f i t s$ to our data. Such fits are made over the correlated $p_{T}^{\mu}-v^{\mu}$ plane with 30 degrees of freedom, giving the following $\chi^{2}$ values: QPM line $\left(\Delta \beta=0.2, R_{s}=1.35\right) x^{2}=54$, EMC line $x^{2}=46$, SLAC line $x^{2}=29$. For comparison, a flat line representing no nuclear effects gives $x^{2}=34$. The two latter fits are acceptable whereas the EMC 1 ine and the QPM 1 ine are disfavoured by $x^{2}$ differences equivalent to 4.10 and $5.0 \sigma$ respectively.

6. DIFFERENTIAL CROSS SECTIONS: $x, y$ and $Q^{2}, w^{2}$ VARTABLES

Secondly we compare the $x$ and $y$ distributions for various regions of $Q^{2}$ and $W^{2}$. Measurement of these variables depends on estimating the total energy of the hadron shower. Since the detection efficiency for neutral hadrons $\left(\pi^{\circ}, n\right)$ is different in $D_{2}$ and Ne all such hadrons were removed from both samples in order to minimize systematic errors. We then correct for the missing hadronic energy using,

with

$$
E_{\bar{v}}=p_{L}^{\mu}+p_{L}^{H} \cdot C
$$

$$
C=\left\langle\mathrm{p}_{\mathrm{T}}^{\mu}\right\rangle /\left\langle\mathrm{p}_{\mathrm{T}}^{\mathrm{H}}{ }_{\mu \nu},\right.
$$

where $\mu$ and $H$ refer to the muon and the charged hadron shower, $L$ and $T$ refer to the longitudinal and tranverse momentum components, respectively, and $\mu \nu$ to the component in the $\mu \nu$ plane. Experimentally, $C=1.651 \pm 0.012$ for $D_{2}$ and $C=1.719 \pm 0.009$ for Ne. The effect of smearing due to this energy correction has been evaluated using a detailed Monte-Carlo simulation of the experimental conditions, and checked by using alternative energy corrections. This effect is small for $x<0.6$, as illustrated in fig. 3, for each of the model assumptions made in sect. 3 . We exclude the region $x>0.6$ from our fit since Fermi motion effects make the application of our models to this region inappropriate. 
The resulting ratios of the $x$ and $y$ distributions in $\mathrm{Ne}$ and $\mathrm{D}_{2}$, for all $Q^{2}$ and for $Q^{2}>4.5 \mathrm{GeV}^{2}$ are shown in $\mathrm{fig} .4$, and Table 2 . The statistical and systematic errors and the average $Q^{2}$ values relevant for each bin are also given in table 2 .

The lines in fig. 4 represent the application of the models described in sect. 3 to antineutrino scattering, accounting for our experimental conditions. The EMC and SLAC lines are not modified by the transition from electroproduction to antineutrino scattering, due to the assumption that they are functions of $x$ only. The departure of these (type A) model predictions from flatness in $y$ is a measure of the correlations imposed by our experimental conditions. By contrast, the QPM model (type B) predicts stronger effects in antineutrino interactions than in electroproduction, and a strong correlation between the behaviour of the $x$ and $y$ distributions. The QPM lines in fig. 4 illustrate these expectations.

Fits were made to the data over the correlated $x-y$ plane with free normalisation as before. Table 3 summarizes the results for various cuts made on $Q^{2}, x$ and $W^{2}$. As for the muon variables, both the SLAC line and the assumption of no nuclear effects give acceptable fits, whereas the EMC Iine and the QPM Iine are disfavoured by 3.90 and 5.60 respectively, when no cuts are made on the data. The consistency of these results with those obtained using the muon variables checks the reliability of the energy correction and the small size of the low topology scan efficiency corrections, which act oppositely in the $\mathrm{p}_{\mathrm{T}}^{\mu}$ and $\mathrm{x}$ variables.

Since the sea quark contribution to the cross section is most reliably determined in $\bar{v}$ interactions, we also considered it valuable to fit the $Q P M$ with $\triangle \beta$ and $R_{S}$ as free parameters. We obtain a good fit, illustrated by the dashed double dot line in fig. 4, with $\Delta \beta=0.16 \pm 0.10$, and $R_{S}=0.91 \pm 0.06$, a decrease rather than an increase in sea content, 70 away from the value, $R_{S}=1.35$, favoured by the QPM interpretation of the EMC data. 
Shadowing in electroproduction $[10,11]$ has been proposed to explain the difference between the SLAC and EMC data at small $x$, since the average $Q^{2}$ of the SLAC data at small $x$ is very low [2]. The average $Q^{2}$ of our uncut data sample is also low. However, our data covers a larger $Q^{2}$ range at sma11 $x$ than the SLAC data and comparison of the uncut data sample with the sample for which $Q^{2}>4.5 \mathrm{GeV}^{2}$ (in fig. 4 and table 2) indicates that the differential cross section ratios show no significant $Q^{2}$ dependence.

To investigate the $Q^{2}$ dependance further we made fits to the correlated $x-y$ plane for various $Q^{2}$ cuts (table 3). A slight decrease in the sea is favoured up to $\left\langle Q^{2}\right\rangle=7.9 \mathrm{GeV}^{2}$ at $x=0.075$. Moreover, removing the region of strongest shadowing $(x<0.1)$ from the fits, still gives no increase in sea since the predicted $r$ ise in the ratio of the $y$ distributions is not observed. Making $w^{2}$ cuts to exclude quasi elastic processes also has no significant effect.

Finally, fig. 5 shows our data plotted directly as a function of $Q^{2}$ for various $x$ ranges. We see that the data show no $Q^{2}$ dependence over the whole $Q^{2}$ range from $0.25 \mathrm{GeV}^{2}$ to $26 \mathrm{GeV}^{2}$.

\section{CONCLUSTONS}

We have made a precise comparison of $\bar{v}$ Ne and $\bar{v} D_{2}$ interactions under identical experimental conditions. The differential cross section ratios have been compared with EMC muon data and SLAC electron data using various models. We find that our data are compatible with the higher $Q^{2}$ EMC data in the medium $x(.3<x<.6)$ region, but they are in disagreement with the rise at small $x(x<.15)$ observed in that data. By contrast, we are in agreement with the lower $Q^{2}$ data of SLAC for all $x$.

Our data show no evidence for $Q^{2}$ dependence at any $x$, in particular there is none at small $x$ in the $Q^{2}$ range $0.25 \mathrm{GeV}^{2}$ to $14 \mathrm{GeV}^{2}$. Thus if shadowing is invoked to explain the behaviour of the $\bar{v}$ and $e^{-}$data at 
small $x$ then shadowing must extend to higher $x$ and higher $Q^{2}$ than expected $[10,11,12]$ and show no $Q^{2}$ dependence in our $Q^{2}$ range.

However, we note that coherent effects $[13,14]$ may cancel shadowing effects at very low $Q^{2}\left(Q^{2}<1 \mathrm{GeV}^{2}\right)$.

We have no evidence for any increase in the sea content of heavy nuclei, in fact our fit results suggest a small decrease, independent of $\mathrm{Q}^{2}$, in agreement with the neon/hydrogen comparison of the BEBC TST [5] and the iron/hydrogen comparison of CDHS [15].

\section{Acknowlegements}

We would like to thank the BEBC, SPS and EMI crews and our scanning and measuring teams for their indispensable contributions to our work. 


\section{REFERENCES}

[1] J.J. Aubert et a1., Phys. Lett. 123B (1983) 275.

[2] (a) A. Bodek et al., Phys. Rev. Lett. 50 (1983) 1431;

(b) A. Bodek et al., Phys. Rev. Lett. 51 (1983) 534;

(c) S. Stein et al., Phys. Rev. D12, (1975) 1884;

(d) D.0. Ca1dwell et a1., Phys. Rev. Lett. 42 (1979) 553.

(e) R.G.Arnold et al., Phys. Rev. Lett. 52 (1984) 727.

[3] A. Bodek and J.L. Ritchie, Phys. Rev. D23 (1981) 1070;

A. Bodek and J.L. Ritchie, Phys. Rev. D24 (1981) 1400.

[4] (a) J.V. Noble, Phys. Rev. Lett. 46 (1981) 412;

(b) J. Szwed, Phys. Lett. 128B (1983) 245;

(c) M.J. Pirner and J.P. Vary, University of Heidelberg UNI-HE-83-02;

(d) S. Date, Wazda University WV-HEP-83-4.

(e) R.L. Jaffe, Phys. Rev. Lett. 50 (1983) 228;

(f) N.N. Nikolaev and Z.I. Zakharov, Phys. Lett. 55B (1975) 397;

(g) C.H. LLewe1lyn Smith, Phys. Lett. 128B (1983) 107;

(h) M. Ericson and A.W. Thomas, Phys. Lett. 128B (1983) 112;

(i) R.M. Godbole and K.V.L. Sarma, Phys. Rev. D25 (1982) 120;

(j) E.L. Berger et a1., ANL-HEP-PR-83-24;

(k) W. Furmanski and A. Kryzwicki, LPTHE 83-11 and Brighton Conference HEP Conference 83-0319.

(l) M. Staszel et al., University of Warsaw, IFT/9/83;

(m) F.E. Close, R.G. Roberts and G.G. Ross, Phys. Lett. 129B (1983) 346;

R.L. Jaffe, F.E. Close, R.G. Roberts and G.G. Ross, Phys. Lett. 134B (1984) 449;

(n) H. Faissner and B.R. Kim, Phys. Lett. 130B (1983) 321;

(o) J. Cleymans and J. Geris, KUL-TF-83-29;

(p) J. Dias de Deus, MPI/PTH 61/83;

(q) O. Nachtmann and H.J. Pirner Z. Phys. C21 (1984) 277.

[5] M.A. Parker et al., Nucl. Phys. B232 (1984) 1.

[6] A.E. Asratyan et a1., ITEP-83-110;

[7] E. Heijne, Yellow Report CERN/83-6.

[8] D. Allasia et a1., Phys. Lett. 135B (1984) 231.

[9] M. Jonker et al., Phys. Lett. 109B (1982) 133. 


\section{REFERENCES (Cont'd)}

[10] M.S. Goodman et al., Phys. Rev. Lett. 47 (1983) 293;

M.T. Miller et al., co0-3065-395.

[11] J.J. Aubert et al., CERN/SPSC 82-4.

[12] A.H. Mueller, Columbia preprint CU-TP-232;

K. Borer et al., Phys. Lett. 30B (1969) 572.

[13] T.H. Bauer et al., Rev. Mod. Phys. 50 (1978) 261;

C.A. Piketty and L. Stodolsky, Nucl. Phys. B15 (1970) 571.

[14] P. Marage et al., IIHE, Brusse1s, preprint 10-83.

[15] J.P. Merlo, CDHS Contribution to Brighton Conference. 


\section{TABLE CAPTIONS}

Table 1 The ratios between isoscalar Ne and $D_{2}$ cross sections per nucleon as a function of $p_{T}^{\mu}$ and $v^{\mu}$, with their statistical and systematic errors and the average $x$ and $Q^{2}$ appropriate to each bin. The systematic errors refer to the shape and not the normalisation of the data.

Table 2 The ratios between isoscalar $\mathrm{Ne}$ and $\mathrm{D}_{2}$ cross sections per nucleon as a function of $x$ and $y$, with their statistical and systematic errors and the average $Q^{2}$ appropriate to each bin, (a) for all $\mathrm{Q}^{2}$, (b) for $Q^{2}>4.5 \mathrm{GeV}^{2}$. The systematic errors refer to the shape and not the normalization of the data.

Table 3 The results of the fits of

$\frac{d^{2} \sigma}{d x d y}(\bar{v} \mathrm{Ne}) / \frac{d^{2} \sigma}{d x d y} \quad\left(\bar{v} D_{2}\right)$

to the EMC, SLAC and $Q P M$ lines ( $\triangle \beta$ and $R_{S}$ fixed and free) described in the text. 
TABLE 1

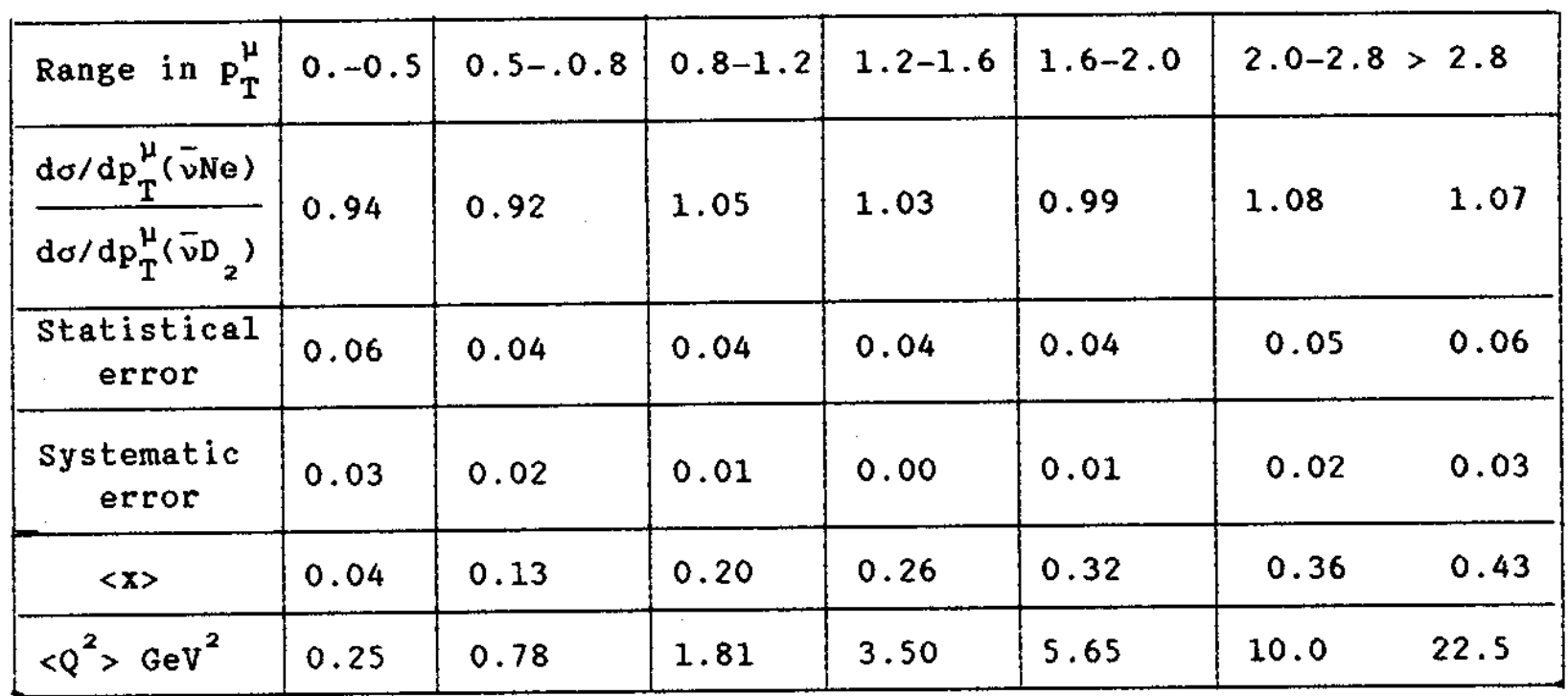

\begin{tabular}{|c|c|c|c|c|c|c|}
\hline Range in $\mathrm{V}^{\mu}$ & $0 .-.02$ & $.02-.04$ & $.04-.06$ & $.06-.08$ & $.08-.15$ & $>0.15$ \\
\hline$\frac{\mathrm{d} \sigma / d v^{\mu}\left(\bar{v}_{\mathrm{Ne}}\right)}{\mathrm{d \sigma} / \mathrm{dv^{ \mu }}\left(\overline{v D}_{2}\right)}$ & 1.00 & 0.96 & 1.00 & 1.12 & 1.05 & 1.03 \\
\hline $\begin{array}{l}\text { Statistical } \\
\text { error }\end{array}$ & 0.04 & 0.04 & 0.04 & 0.06 & 0.04 & 0.06 \\
\hline $\begin{array}{c}\text { Systematic } \\
\text { error }\end{array}$ & 0.02 & 0.01 & 0.01 & 0.01 & 0.01 & 0.02 \\
\hline$\langle x\rangle$ & 0.12 & 0.20 & 0.25 & 0.27 & 0.33 & 0.45 \\
\hline$\left\langle Q^{2}\right\rangle \mathrm{GeV}^{2}$ & 0.83 & 2.14 & 3.85 & 5.30 & 8.17 & 16.3 \\
\hline
\end{tabular}


TABLE 2(a): All $Q^{2}$

\begin{tabular}{|c|l|l|l|l|l|lc|}
\hline Range in $x$ & $0 .-.05$ & $.05-.1$ & $.1-.2$ & $.2-.3$ & $.3-.45$ & $.45-.6$ & $.6-.1$ \\
\hline$\frac{\mathrm{d} \sigma / \mathrm{dx}(\overline{\mathrm{uNe}})}{\mathrm{d \sigma} / \mathrm{dx}\left(\overline{\mathrm{v}} \mathrm{D}_{2}\right)}$ & 1.01 & 0.97 & 1.01 & 1.13 & 0.99 & 0.97 & 1.04 \\
\hline $\begin{array}{c}\text { Statistical } \\
\text { error }\end{array}$ & 0.05 & 0.04 & 0.04 & 0.05 & 0.04 & 0.06 & 0.07 \\
\hline $\begin{array}{c}\text { Systematic } \\
\text { error }\end{array}$ & 0.03 & 0.03 & 0.03 & 0.02 & 0.03 & 0.05 & 0.09 \\
\hline $\mathrm{Q}^{2}>\mathrm{GeV}^{2}$ & 1.07 & 2.59 & 4.33 & 6.14 & 7.67 & 8.28 & 6.35 \\
\hline
\end{tabular}

\begin{tabular}{|c|c|c|c|c|c|c|}
\hline Range in $y$ & $0 .-.1$ & $.1-.2$ & $.2-.4$ & $.4-.6$ & $.6-.8$ & $.8-.1$ \\
\hline$\frac{\mathrm{do} / \mathrm{dy}(\overline{\mathrm{vNe}})}{\mathrm{do} / \mathrm{dy}\left(\overline{\mathrm{v}} \mathrm{D}_{2}\right)}$ & 0.98 & 1.00 & 1.05 & 1.03 & 1.01 & 0.96 \\
\hline $\begin{array}{c}\text { Statistical } \\
\text { error }\end{array}$ & 0.04 & 0.04 & 0.03 & 0.04 & 0.06 & 0.10 \\
\hline $\begin{array}{l}\text { Systematic } \\
\text { error }\end{array}$ & 0.04 & 0.02 & 0.02 & 0.03 & 0.04 & 0.06 \\
\hline$\left\langle Q^{2}\right\rangle \mathrm{GeV}^{2}$ & 1.92 & 3.09 & 4.75 & 7.18 & 9.42 & 14.21 \\
\hline
\end{tabular}


TABLE 2(b): $Q^{2}>4.5 \mathrm{GeV}^{2}$

\begin{tabular}{|c|l|l|l|l|l|l|}
\hline Range in $\mathrm{x}$ & $.05-.1$ & $.1-.2$ & $.2-.3$ & $.3-.45$ & $.45-.6$ & $.6-1$. \\
\hline$\frac{\mathrm{d \sigma} / \mathrm{dx}(\overline{\mathrm{uNe}})}{\mathrm{d} \sigma / \mathrm{dx}\left(\bar{v}_{2}\right)}$ & 0.91 & 1.02 & 1.09 & 1.00 & 0.92 & 1.07 \\
\hline $\begin{array}{c}\text { Statistical } \\
\text { error }\end{array}$ & 0.11 & 0.07 & 0.07 & 0.07 & 0.08 & 0.11 \\
\hline $\begin{array}{c}\text { Systematic } \\
\text { error }\end{array}$ & 0.03 & 0.03 & 0.02 & 0.03 & 0.03 & 0.07 \\
\hline $\mathrm{QQ}^{2}>\mathrm{GeV}^{2}$ & 7.9 & 9.5 & 11.5 & 13.2 & 13.9 & 11.6 \\
\hline
\end{tabular}

\begin{tabular}{|c|l|l|l|l|l|l|}
\hline Range in $\mathrm{y}$ & $0 .-.1$ & $.1-.2$ & $.2-.4$ & $.4-.6$ & $.6-.8$ & $.8-1$. \\
\hline$\frac{\mathrm{d} \sigma / \mathrm{dy}(\overline{\mathrm{v} N e})}{\mathrm{d} \sigma / \mathrm{dy}\left(\overline{\mathrm{u}} \mathrm{D}_{2}\right)}$ & 1.13 & 0.98 & 1.10 & 0.96 & 0.99 & 0.93 \\
\hline $\begin{array}{c}\text { Statistical } \\
\text { error }\end{array}$ & 0.15 & 0.09 & 0.06 & 0.06 & 0.07 & 0.12 \\
\hline $\begin{array}{c}\text { Systematic } \\
\text { error }\end{array}$ & 0.03 & 0.02 & 0.02 & 0.03 & 0.03 & 0.04 \\
\hline $\mathrm{QQ}^{2}>\mathrm{GeV}^{2}$ & 7.8 & 8.6 & 9.9 & 18.1 & 14.0 & 18.8 \\
\hline
\end{tabular}




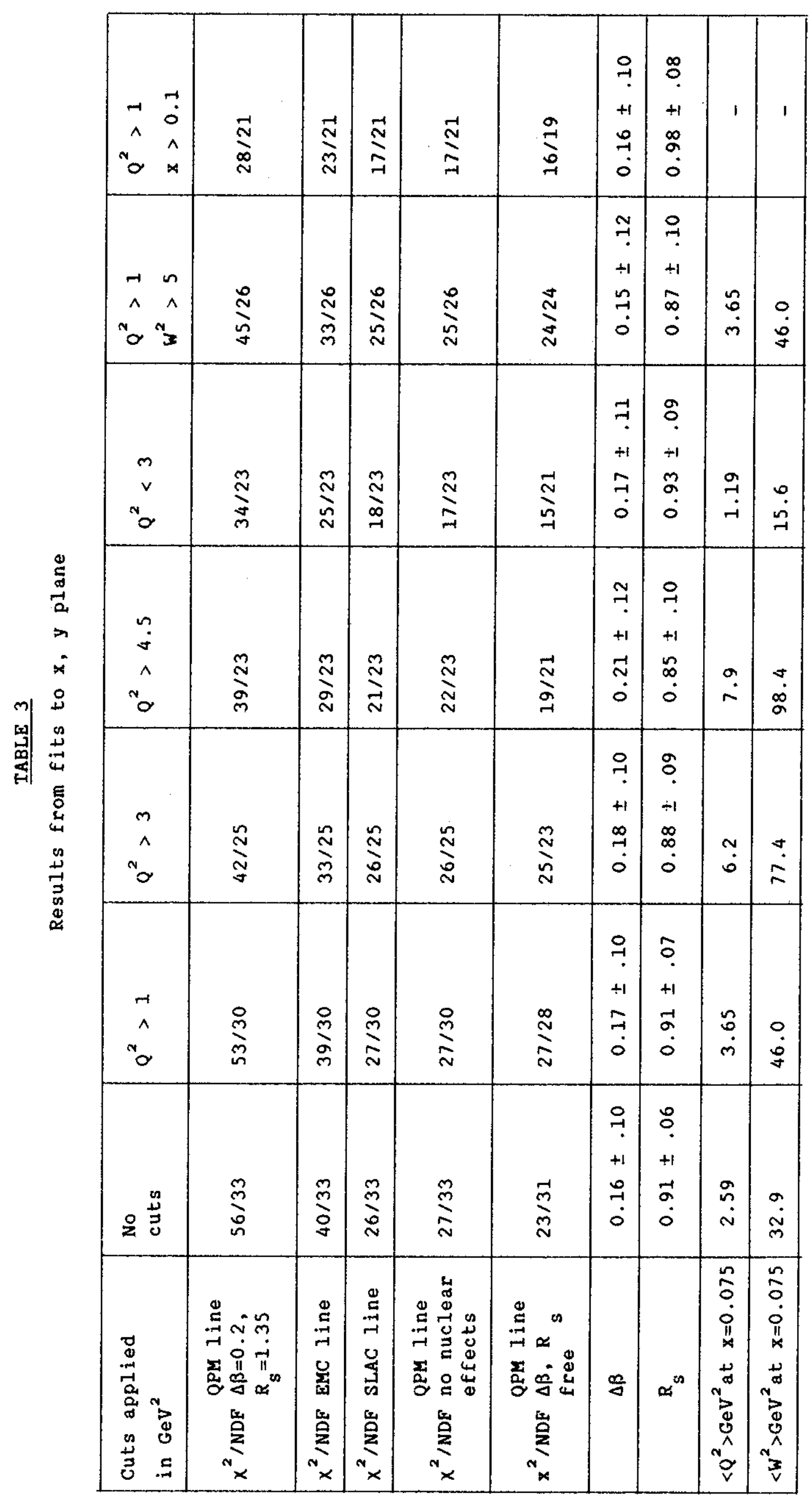




\section{FIGURE CAPTIONS}

Fig. 1 The ratio of isoscalar muoproduction and electroproduction cross sections between heavy and light nuclear targets as a function of $x$ : (a) $0, \mu \mathrm{Fe} / \mu \mathrm{D}_{2}$ EMC data [11], (the shading indicates the possible systematic shape error in these data); (b) $0, \Delta, \mathbf{A}$, eAl/eD 2 SLAC data $[2 b],[2 c],[2 d],[2 e]$ respectively. The dashed dot line is the fit to their data given by EMC, the dashed line is our representation of the SLAC $\mathrm{Al} / \mathrm{D}_{2}$ data (we have averaged the differing normalisations of the SLAC data), and the full line represents the quark parton model (QPM) explained in the text for $\triangle B=0.2$ and $R_{S}=1.35$. The $E M C$ and $Q P M$ lines are nearly coincident on this figure.

Fig. 2 The ratio between isoscalar Ne and $D_{2} \bar{v}$ cross sections per nucleon as a function of: (a) $p_{T}^{\mu} ;$ (b) $v^{\mu}$. The error bars include both statistical and systematic errors. The overlaid lines are the SLAC (dashed), EMC (dashed-dot) and QPM (full) lines of $\mathrm{fig}$. 1 translated to these muon variables.

Fig. 3 The ratio between the predicted ratios of the isoscalar Ne and $\mathrm{D}_{2} \bar{v}$ cross section per nucleon as a function of $\mathrm{x}$, before and after hadron energy smearing, as evaluated by Monte-Carlo, for each of the models described in sect. 3. The key to the lines is given in fig. 1 .

Fig. 4 The ratio between isoscalar Ne and $D_{2} \bar{v}$ cross sections per nucleon as a function of $x(a, c)$ and $y(b, d)$, for all $Q^{2}(a, b)$ and $Q^{2}>4.5 \mathrm{GeV}^{2}$ (c, d). The error bars include both statistical and systematic errors. The overlaid lines are the SLAC (dashed), EMC (dashed-dot) and QPM (full) lines of fig. 1 translated to these variables, with the addition of the QPM (dashed-double-dot) line for the best fit values $\Delta \beta=0.16$, $\mathrm{R}_{\mathrm{s}}=0.91(\mathrm{a}, \mathrm{b})$ and $\Delta \beta=0.21, \mathrm{R}_{\mathrm{s}}=0.85(\mathrm{c}, \mathrm{d})$.

Fig. 5 The ratio between isoscalar $\mathrm{Ne}$ and $D_{2} \bar{v}$ cross sections per nucleon as a function of $Q^{2}$, for the $x$ ranges, $x<0.15$, $0.15<x<0.4, x>0.4$. The error bars include both statistical and systematic errors. The overlaid lines are the SLAC (dashed), EMC (dashed-dot) and QPM (full) lines of fig. 1 translated to these variables. 


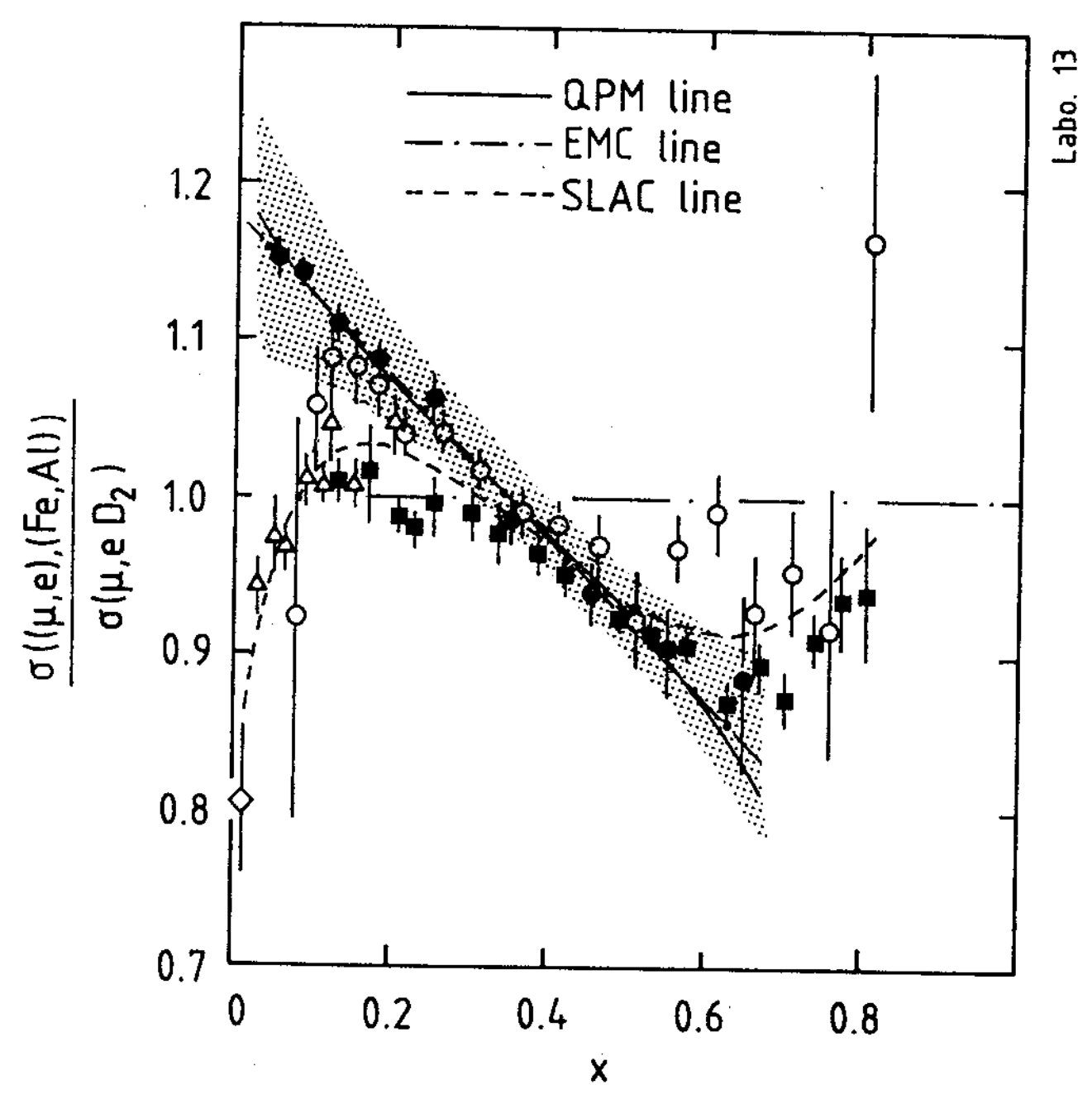

Fig. 1 


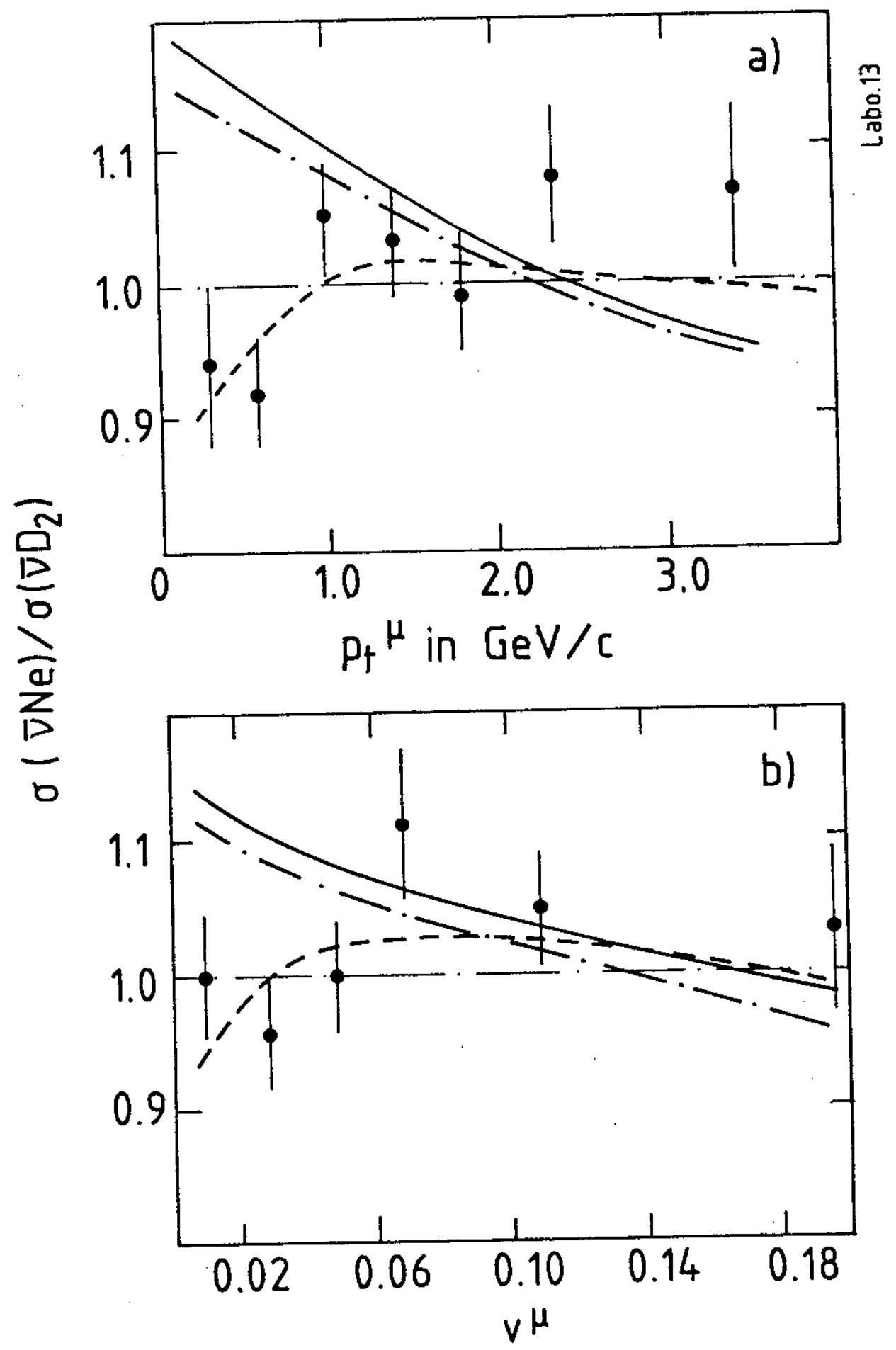

Fig. 2 


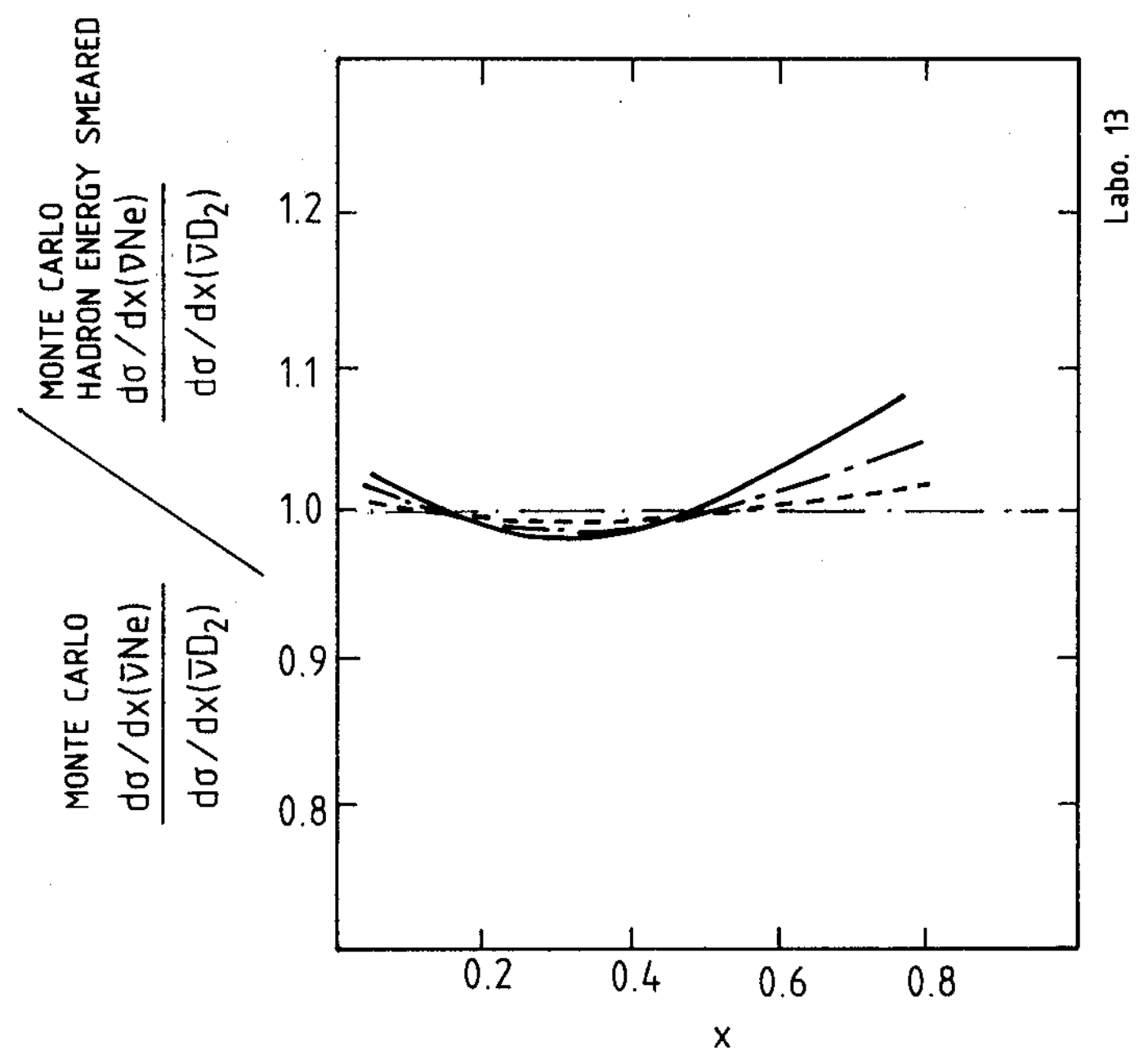

Fig. 3 


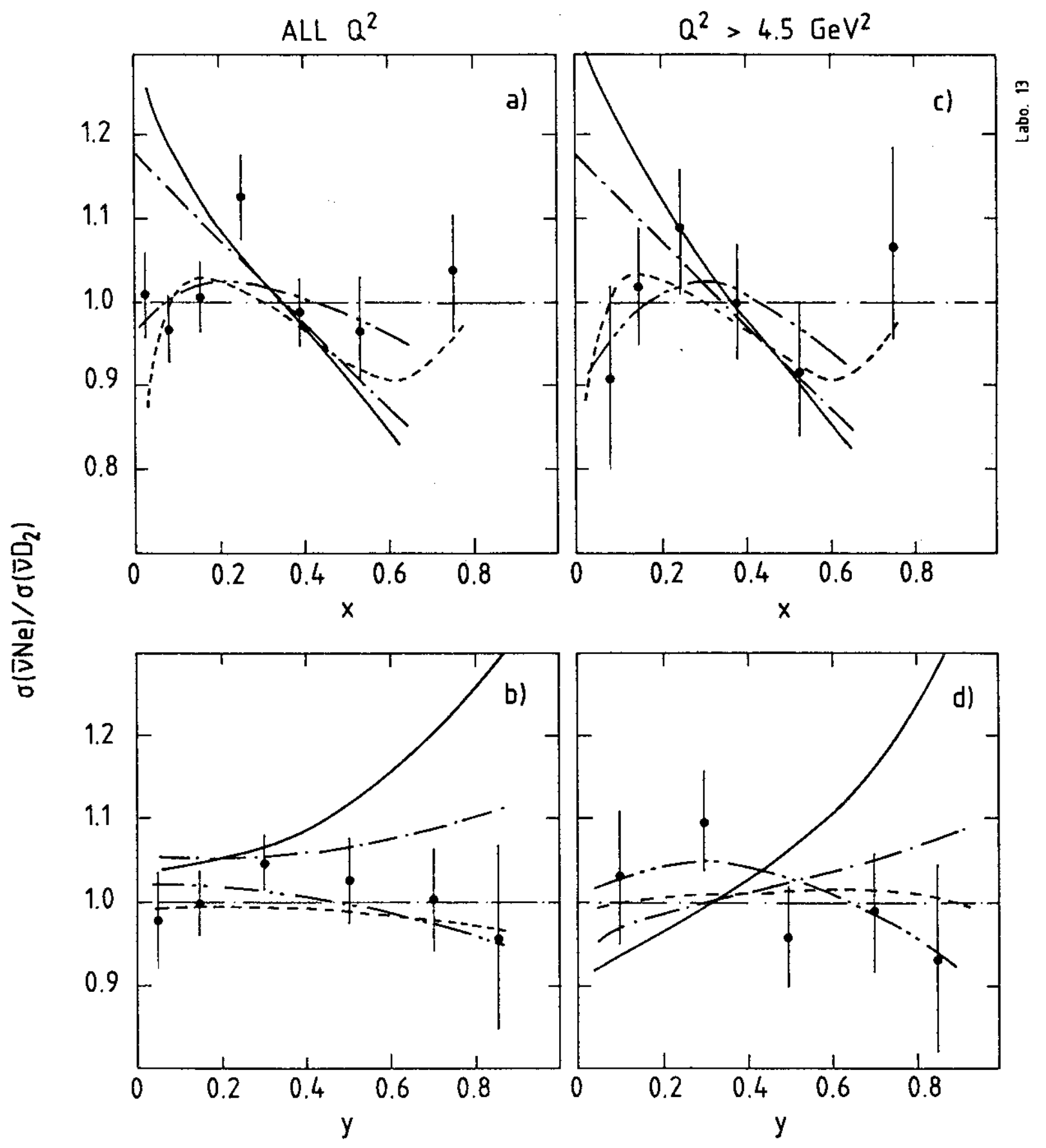

Fig. 4 


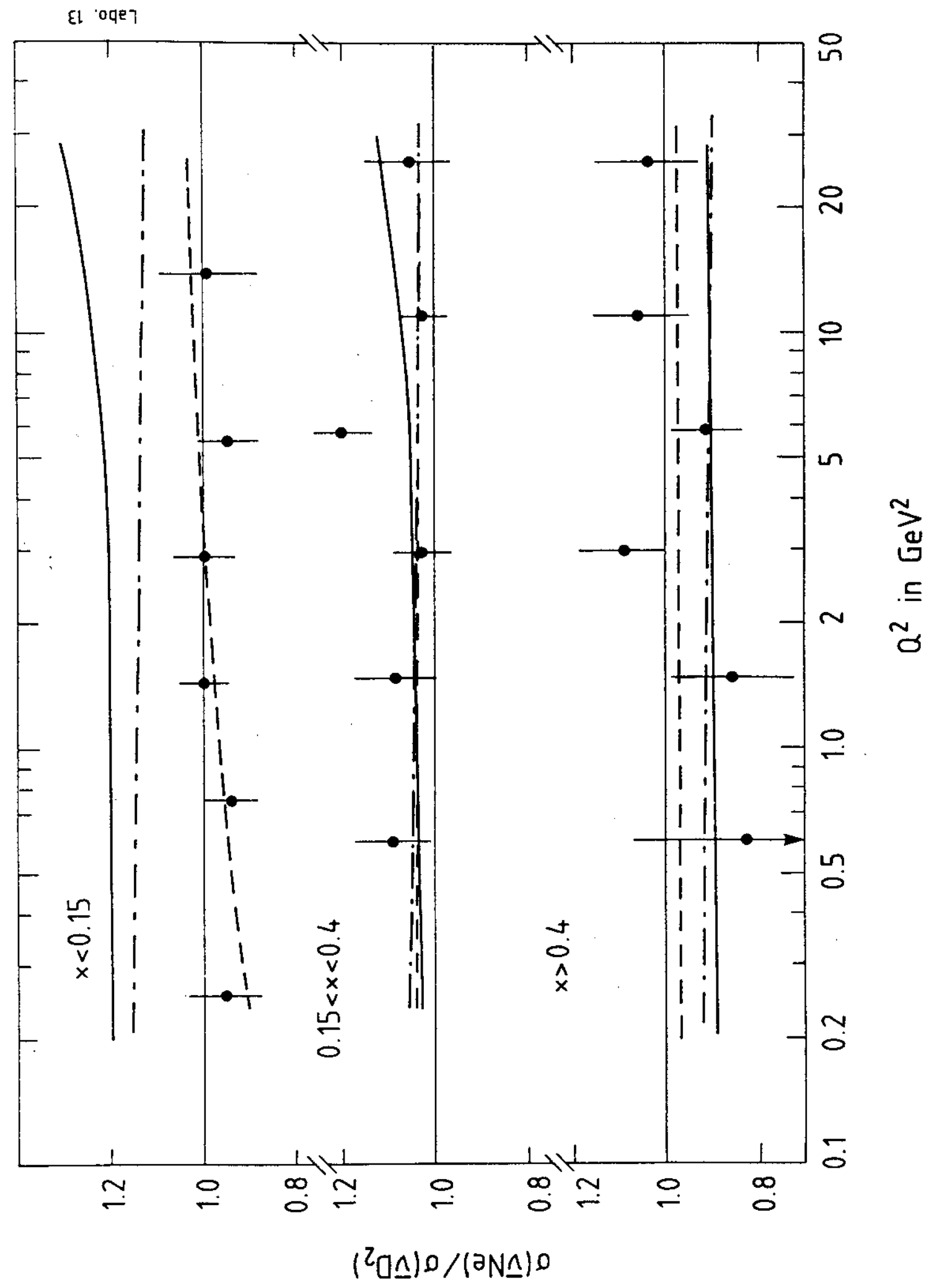

Fig. 5 